Bull. Austral. Math. Soc.

$13 \mathrm{~B} 20,13 \mathrm{G} 05$

Vol. 37 (1988) [353-366]

\title{
INTEGRALLY CLOSED FACTOR DOMAINS
}

\author{
Valentina Barucci, David E. Dobbs and S.B. Mulay
}

\begin{abstract}
This paper characterises the integral domains $R$ with the property that $R / P$ is integrally closed for each prime ideal $P$ of $R$. It is shown that Dedekind domains are the only Noetherian domains with this property. On the other hand, each integrally closed goingdown domain has this property. Related properties and examples are also studied.
\end{abstract}

\section{INTRODUCTION}

Let $R$ be a (commutative integral) domain. It is well-known that $R$ is integrally closed if and only if each localisation $R_{P}$ is integrally closed. Our interest here is to ask the "dual" question: for which $R$ is it the case that $R / P$ is integrally closed for all $P \in \operatorname{Spec}(R)$ ? Not all integrally closed domains $R$ have this property, as we see by considering (see [13, (3), page 262]) $R=D[X, Y]$ and $P=\left(X^{2}-Y^{3}\right)$, where $D$ is any integrally closed domain. Notice in this example that $R$ is Noetherian, and of (Krull) dimension at least 2 , if $D$ is also assumed Noetherian. However, there are instances in which the property in question holds. For instance, if $R$ is a Prüfer domain, then each factor domain $R / P$ is also a Prüfer domain and, hence, is integrally closed. Also, if $R$ is integrally closed and $\operatorname{dim}(R) \leqslant 1$, then each factor domain of $R$ is either $R$ or a field, and hence is integrally closed.

The two main results of this paper generalise the above examples. The first of these, Theorem 3.1, asserts that if $R$ is an integrally closed Noetherian domain of dimension at least 2 , then $R / P$ is not integrally closed for at least one height 1 prime ideal $P$ of $R$. The second main result, Theorem 4.1, concerns going-down domains (in the sense of [4]). Recall that arbitrary Prüfer domains and arbitrary domains of dimension at most 1 are examples of going-down domains. Theorem 4.1 asserts that if $R$ is an integrally closed going-down domain, then so is $R / P$, for each $P \in \operatorname{Spec}(R)$. Theorems 3.1 and 4.1 thus give a new way in which Noetherian domains and going-down domains manifest "opposite" behaviour. Another way is via their spectra. Indeed, if $R$ is a going-down domain, then $\operatorname{Spec}(R)$ as a poset under inclusion is a tree [4, Theorem

Received 22 July 1987

The authors were supported in part by a NATO Collaborative Research Grant. Dobbs was also supported in part by the Consiglio Nazionale delle Ricerche and the UT Science Alliance. He thanks the Università di Roma, "La Sapienza", for its warm hospitality.

Copyright Clearance Centre, Inc. Serial-fee code: $0004-9729 / 88 \$ \$ 2.00+0.00$. 
2.2]; while Krull's principal ideal theorem implies, for a Noetherian domain $R$, that $\operatorname{Spec}(R)$ is a tree if and only if $\operatorname{dim}(R) \leqslant 1$.

We next describe the organisation of this paper. Section 2 is concerned with generalities about the property in question, including a characterisation of it in Proposition 2.3. Here and elsewhere, the CPI-extension of $R$ with respect to $P$ (in the sense of [1]) and other pullbacks intervene naturally. Section 3 is devoted to the proof of Theorem 3.1. Using the material in Section 2, we reduce this to the case of a two-dimensional local (Noetherian) domain $(R, M)$. This case is then attacked by treating separately the subcases of finite or infinite residue field $R / M$. The proof for the latter subcase retains a faint image of the motivating $X^{2}-Y^{3}$ example. In addition to establishing Theorem 4.1, Section 4 studies the passage, from a going-down domain $R$ to a factor domain $R / P$, of properties that are less restrictive than "integrally closed," such as "root closed" and "seminormal." For this work, the characterisation of going-down domains in terms of divided domains [5] is a useful tool.

\section{ChaRACTERISATIONS}

To state many of our results, it will be convenient to let $\underline{C}$ denote the class of commutative rings $A$ such that $A / P$ is integrally closed for each $P \in \operatorname{Spec}(A)$. As noted in the introduction, $\underline{C}$ contains each Prüfer domain and each integrally closed domain of dimension at most 1 . Proposition 2.1(b) will explain how the study of $C$ is reduced to determining which domains are in $\underline{C}$.

Proposition 2.1. Let $A$ be a commutative ring. Then:

(a) $A \in \underline{C} \Longleftrightarrow A_{P} \in \underline{C}$ for each $P \in \operatorname{Spec}(A) \Longleftrightarrow A_{M} \in \underline{C}$ for each $M \in \operatorname{Max}(A)$;

(b) $A \in \underline{C}: \Longleftrightarrow A / P \in \underline{C}$ for each minimal (that is, height 0 ) prime $P$ of $A$;

(c) $A \in \underline{C} \Longleftrightarrow A_{\text {red }} \in \underline{C}$;

(d) If $\operatorname{dim}(A)=\mathbf{0}$ (for instance, if $A$ is von Neumann regular), then $A \in \underline{C}$.

Proof: (a) Suppose $A \in \underline{C}$, and consider $P \in \operatorname{Spec}(A)$. To show $A_{P} \in \underline{C}$, we need to prove that $D=A_{P} / Q A_{P}$ is integrally closed for all primes $Q \subset P$ of $A$. To do this, take $S=\{a+Q \in A / Q: a \in A \backslash P\}$, and note that $D \cong S^{-1}(r \backslash Q)$ is a ring of fractions of the integrally closed domain $A / Q$.

Conversely, suppose that $A_{M} \in \underline{C}$ for all $M \in \operatorname{Max}(A)$. To show $A \in \underline{C}$, we consicler a prime $P$ of $A$ and must show that $A / P$ is integrally closed. We shall show, equivalently, that $E=(A / P)_{M / P}$ is integrally closed for all maximal ideals $M$ containing $P$. To do this, note that $E \cong A_{M} / P A_{M}$, a factor domain of a domain in C.

(b) Since every prime $Q$ of $A$ contains a minimal prime $P$, the assertion follows from the standard isomorphism $(A / P) /(Q / P) \cong A / Q$. 
(c) A direct proof as in (b) is possible. Instead, we shall use (b). It suffices to note that the minimal primes of $A_{\text {red }}$ have the form $P_{1}=P / \sqrt{A}$, where $P$ is any minimal prime of $A$; and that $A / P \cong A_{\text {red }} / P_{1}$.

(d) This is evident since each factor domain of a zero-dimensional ring is a field, and hence integrally closed. For a more esoteric proof, use (c) and (a), noting that $A_{P}$ is a field (and hence in $\underline{C}$ ) for each prime $P$ of a von Neumann regular ring $A$.

The next example shows that domains in $\underline{C}$ other than those mentioned above can have Prüferian proper factor domains.

Example 2.2. There exists an (integrally closed) domain $R$ in $\underline{C}$ such that $R / P$ is a Prüfer domain for each nonzero $P \in \operatorname{Spec}(R), R$ is not a Prüfer domain, and $\operatorname{dim}(R)=2$.

For the construction, we use data satisfying the following conditions: $(S, M)$ is a quasilocal one-dimensional nonvaluation domain with residue field $k=S / M$ and $V$ is a one-dimensional valuation ring of $k$. (For instance, take $S=\mathbf{Q}+X \mathbf{Q}(Y)[[X]]$ and $V=\mathbb{Z}_{2 Z}$.) Letting $\varphi: S \rightarrow k$ denote the canonical surjection, we assert that the pullback $R=\varphi^{-1}(V)$ has the stated properties.

Indeed, standard pullback methods (see [8, Theorem 1.4 and Corollary 1.5(5)]) yield that $R$ is an integrally closed two-dimensional quasilocal domain whose only nonzero nonmaximal prime ideal is $M$. Observe that $R / M \cong V$ is (a valuation and hence) a Prüfer domain. Finally, note that $R$ is not a Prüfer domain, since $S$ is a quasilocal nonvaluation overring of $R$.

In view of the diversity suggested above, it will be helpful next to give a characterisation of the domains in $\underline{C}$. First, recall from[1], $[7],[8]$ that if $R$ is a domain and $P \in \operatorname{Spec}(R)$, the C:PI-extension of $R$ with respect to $P$ is $R+P R_{P}$, which may be viewed as the pullback $R / P_{X_{R_{P}} / P R_{P}} R_{P}$; and that the contraction map gives a bijection between $\operatorname{Spec}\left(R+P R_{P}\right)$ and $\{Q \in \operatorname{Spec}(R)$ : either $Q \subset P$ or $P \subset Q\}$. To motivate the condition in Proposition 2.3(a), recall the well-known result of E.D. Davis that $R$ is a Prüfer domain if and only if each overring of $R$ is integrally closed.

Proposition 2.3. Let $R$ be a domain. Then:

(a) $R \in \underline{C} \Longleftrightarrow R+P R_{P}$ is integrally closed for all $P \in \operatorname{Spec}(R)$;

(b) let $P \in \operatorname{Spec}(R)$. Then the following six conditions are equivalent:

(i) if $y$ is in the quotient field of $R / P$ and $y^{n}+\sum_{i=0}^{n-1}\left(r_{i}+P\right) y^{i}=0$ for some finite subset $\left\{r_{0}, \ldots, r_{n-1}\right\}$ of $R$, then $y \in R / P$; 
(ii) if $a \in R, b \in R \backslash P$ and $a^{n}+\sum_{i=0}^{n-1} r_{i} a^{i} b^{n-i} \in P$ for some finite subset $\left\{r_{0}, \ldots, r_{n-1}\right\}$ of $R$, then $b x-a \in P$ for some $x \in R$;

(iii) = "(ii)" with $a \in R \backslash P$;

(iv) if $a \in R \backslash\{0\}, b \in R \backslash P$ and $a=p\left(a^{-1}\right)^{n-1}-b\left(\sum_{i=0}^{n-1} r_{i}\left(b a^{-1}\right)^{n-1-i}\right)$ for some $p \in P$ and a finite subset $\left\{r_{0}, \ldots, r_{n-1}\right\}$ of $R$, then $a \in(P, b)$;

(v) = "(iv)" with $a \in R \backslash P$;

(vi) $R / P$ is integrally closed.

\section{Proof:}

(a) If follows from a proof of Greenberg [10, Proposition 4.7] that if $P \in \operatorname{Spec}(R)$, then $R+R P_{P}$ is integrally closed if and only if both $R_{P}$ and $R / P$ are integrally closed. However, Greenberg's result assumes, but does not use, the extra hypothesis that $P$ is a flat ideal. So, for the sake of clarity, we next offer a fresh proof of Greenberg's result.

Let $A$ denote the integral closure of $R+P R_{P}$ in $R_{P}$ and let $B$ denote the integral closure of $R / P$. By $[8$, Corollary $1.5(5)], A=B_{x_{R_{P}} / P R_{P}} R_{P}$. If $R / P$ is integrally closed, $B=R / P$ and so $A=R+P R_{P}$ is integrally closed in $R_{P}$. Thus, if both $R / P$ and $R_{P}$ are integrally closed, so is $R+R_{P}$. Conversely, suppose that $R+P R_{P}$ is integrally closed. Hence, so is its localisation at $P R_{P}$. But this localisation is just $R_{P}$, by [7, Lemma 2.2(a)]. Moreover, the hypothesis is that $A=R+P R_{P}$, whence $B=A / P R_{P} \cong R / P$. Thus, $R / P$ is also integrally closed, completing the "fresh proof". We next use this assertion to obtain (a).

Suppose first that $R \in \underline{C}$, and consider $P \in \operatorname{Spec}(R)$. To show that $R+P R_{P}$ is integrally closed, the above result directs us to $R / P$ and $R_{P}$. The former is integrally closed because $R \in \underline{C}$; the latter is integrally closed because $R(\cong R / 0)$ is.

Conversely, suppose that each $R+P R_{P}$ is integrally closed. By the above result, so is each $R / P$; that is, $R \in \underline{C}$.

(b) (i) $\Longleftrightarrow$ (vi): Cllear.

(i) $\Longrightarrow$ (ii): Suppose $a, b$ are as in (ii). Put $y=a b^{-1}+P R_{P}$ in $R_{P} / P R_{P}$, the quotient field of $R / P$. Now, since $b^{-1} \in R_{P}$,

$$
y^{n}+\sum_{i=0}^{n-1}\left(r_{i}+P\right) y^{i}=\left(a^{n}+\sum_{i=0}^{n-1} r_{i} a^{i} b^{n-i}\right) b^{-n}+P R_{P}=P R_{P}
$$

is 0 in $R_{P} / P R_{P}$, and so (i) gives $y \in R / P$. In other words, $a b^{-1}-r \in P R_{P}$ for some $r \in R$. Thus, $a-b r=b\left(a b^{-1}-r\right) \in P R_{P} \cap R=P$, which immediately gives (ii), with $x=r$.

(ii) $\Longrightarrow$ (iii): Trivial.

(iii) $\Longrightarrow$ (ii): If $a \in P$ and $b \in R \backslash P$, any $x \in P$ satisfies $b x-a \in P$. 
(ii) $\Longrightarrow$ (i): Let $y$ be as in (i). Write $y=a b^{-1}+P R_{P}$, for some $a \in R$ and $b \in R \backslash P$. Then $z=a b^{-1}$ satisfies

$$
z^{n}+r_{n-1} z^{n-1}+\cdots+r_{1} z+r_{0} \in P R_{P}
$$

whence, multiplying by $b^{n}$, we have

$$
a^{n}+r_{n-1} a^{n-1} b+\cdots+r_{1} a b^{n-1}+r_{0} b^{n} \in P R_{P} \cap R=P .
$$

By (ii), $b r-a \in P$ for some $r \in R$. Thus, multiplying by $-b^{-1}$, we have $z-r \in P R_{P}$. Hence, $y=z+P R_{P}=r+P R_{P}$ is in $R / P$, proving (i).

(ii) $\Longleftrightarrow$ (iv): Notice that the assertion in (ii) is trivial if $a=0$, by using any $x \in P$. Hence, we may take $a \neq 0$. It now suffices to notice that the equation in (iv) is equivalent to

$$
a^{n}=a^{n-1}\left[p\left(a^{-1}\right)^{n-1}-b\left(\sum_{i=0}^{n-1} r_{i}\left(b a^{-1}\right)^{n-1-i}\right)\right]
$$

that is, to $a^{n}+\sum_{i=0}^{n-1} r_{i} a^{i} b^{n-i}=p$, which is essentially the condition in (ii).

(iii) $\Longleftrightarrow$ (v): repeat the argument for (ii) $\Longleftrightarrow$ (iv), noting that $a$ is now automatically nonzero.

The conditions in Proposition 2.3 will lead to a fast proof of one of our main results, Theorem 4.1. Rather than review the necessary background for it here, we close this section with some assorted remarks.

Remark 2.4. (a) The following result has some of the flavour of the conditions in Proposition 2.3(b). Let $R$ be a domain and $P \in \operatorname{Spec}(R)$. Suppose that $a, b \in R \backslash P$ implies that either $a \mid b$ or $b \mid a$. Then $R / P$ is a valuation domain.

For a proof, delly, and hence take $a, b \in R \backslash P$ such that neither $\bar{a}=a+P$ nor $\bar{b}=b+P$ divides the other in $R / P$. Hence, neither $a$ nor $b$ divides the other in $R$, contrary to hypothesis.

(b) The analogy between (a) and Proposition 2.3(b) is not perfect, for the converse of the result in (a) is false. To see this, consider $R=K[[X, Y]]$, where $K$ is a field, and $P=(X-Y)$. It is possible to show that $R / P \cong K[[X]]$, a (discrete rank 1 ) valuation domain, although $a=X, b=Y$ are in $R \backslash P$ and neither $a$ nor $b$ divides the other.

(c) It is important to note that $R / P$ can be integrally closed without the CPIextension $R+P R_{P}$ reducing to $R$. In other words, $R / P$ being integrally closed does not imply $P=P R_{P}$. To illustrate this, we need only consider a maximal ideal $P$ of a nonquasilocal domain $R$; or a prime $P$ of a Prüfer domain $R$ such that $P$ is not contained in the Jacobson radical of $R$; or $P=(X)$ in $R=K[X, Y]$, where $K$ is a field; or a host of other examples. Despite the evident rarity of the " $P=P R_{P}$ " condition, it will be very useful in the proof of Theorem 4.1 and Proposition 4.4 . 


\section{The Noetherian case}

In this section and the next, we interpret the conditions in Proposition 2.3 for certain classes of domains. For Noetherian domains, the full answer is given in the following main result.

Theorem 3.1. Let $R$ be an integrally closed Noetherian domain. Then:

(a) if $Q \in S_{p e c}(R)$ and $h t(Q)=2$, then there exists $P \in \operatorname{Spec}(R)$ such that $P \subset Q, h t(P)=1$, and $R / P$ is not integrally closed;

(b) if $\operatorname{dim}(R)>1$, then there exists $P \in S p e c(R)$ such that $h t(P)=1$ and $R / P$ is not integrally closed, and so $R \notin \underline{C}$;

(c) $R \in \underline{C} \Longleftrightarrow R$ is a Dedekind domain $\Longleftrightarrow \operatorname{din}(R) \leqslant 1$.

Assume, for the moment, that (a) has been proved. We show next how (b) and (c) are easy consequences.

(b) Choose $N \in \operatorname{Max}(R)$ with $h t(N)>1$. But $h t(N)<\infty$, by a standard consequence [11, Theorem 152] of Krull's principal ideal theorem. Hence $N$ contains a height 2 prime, say $Q$. Apply (a) to $Q$.

(c) Since $R$ is Dedekind if and only if $R$ is (integrally closed Noetherian and) of dimension $\leqslant 1$, it is enough to apply (b) and recall that all domains of dimension $\leqslant 1$ are in $\underline{C}$.

The rest of the argument is devoted to proving (a). First, we reduce the problem to proving the following assertion:

$\left(^{*}\right)$ if $(R, M)$ is a two-dimensional integrally closed local (Noetherian) domain, then $R / P$ is not integrally closed for some height 1 prime $P$ of $R$.

We show next how (a) follows from $\left(^{*}\right)$. This will be done by reworking the proof of Proposition 2.1(a). Let $R, Q$ be as in (a). Consider the two-dimensional integrally closed local Noetherian domain $R_{Q}$. By $\left(^{*}\right)$, it contains a height 1 prime $I$ such that $R_{Q} / I$ is not integrally closed. Since $I=P R_{Q}$ for some height 1 prime $P \subset Q$, it follows that $(R / P)_{Q / P} \cong R_{Q} / I$ is not integrally closed. Hence, neither is $R / P$.

It remains only to prove $\left(^{*}\right)$. For the remainder of this section, let $(R, M)$ be as in (*). There are two cases, determined by whether $k=R / M$ is finite. For each case, it will be helpful to consider

$$
\operatorname{gr}(R)=k \oplus M / M^{2} \oplus M^{2} / M^{3} \oplus \ldots,
$$

the associated graded ring of $R$.

PROOF OF (*) IF $k$ IS INFINITE: Since $M$ is an ideal of definition $R$, it follows from [2, Corollaire, page VIII.66] that $\operatorname{dim}(\operatorname{gr}(R))=\operatorname{dim}(R)=2$. Moreover, $\operatorname{gr}(R)$ is a finite-type $k$-algebra. Indeed, if $\left\{m_{1}, \ldots, m_{n}\right\}$ is a generating set for $M$ as an 
ideal of $R$, then $\operatorname{gr}(R)=k\left[\bar{m}_{1}, \ldots, \bar{m}_{n}\right]$, where $\bar{m}_{i}=m_{i}+M^{2}$ in $M / M^{2}$. Thus, the Noether Normalization Theorem (NNT) gives $X, Y \in \operatorname{gr}(R)$, a pair of elements which are algebraically independent over $k$, such that $\operatorname{gr}(R)$ is integral over $k[X, Y]$. Nowand this is where we use the hypothesis that $k$ is infinite-it follows from the proof of NNT [13, Theorem 8, page 266] that $X, Y$ can be chosen in $\sum k \overline{m_{i}}$. So chosen, $X$ and $Y$ are in $M / M^{2}$. Choose $x, y \in M$ such that $X=x+M^{2}, Y=y+M^{2}$. Since $X, Y$ are linearly independent over $k,\{x, y\}$ is part of a minimal generating set for $M$. In particular, $x y^{-1} \notin R$.

Consider $f=x^{2}-y^{3}$. Note that $f \neq 0$, lest $\left(x y^{-1}\right)^{2}=y \in R$, contradicting the hypothesis that $R$ is integrally (really, 2-root) closed. Thus, if $P \in \operatorname{Spec}(R)$ is chosen minimal over $R f$, the principal ideal theorem gives $h t(P)=1$. If we deny the result, it follows from a conment in the above proof of (c) that $R / P$ is a local Dedekind domain but not a field. In other words, $A=R / P$ is a DVR. Let $N$ denote $M / P$, the maximal ideal of $A$. Notice that the residue field of $A$ is $A / N \cong k$. Since $A$ is a one-dimensional regular local ring [14, Theorem 25 , page $30 \mathrm{~T}]$ yields that $\operatorname{gr}(A) \cong k[T]$ as $k$-algebras, for some indeterminate $T$ over $k$. Also, [14, Theorem 2, page 250] gives that $\operatorname{gr}(A)$ is isomorphic to the graded module associated to the $R$-module $A$. In other words,

$$
\operatorname{gr}(A) \cong \bigoplus_{n=0}^{\infty} M^{n} A / M^{n+1} A=\bigoplus_{n=0}^{\infty}\left[\left(M^{n}+P\right) / P\right] /\left[\left(M^{n+1}+P\right) / P\right]
$$

that is, we identify $\operatorname{gr}(A) \cong \oplus\left(M^{n}+P\right) /\left(M^{n+1}+P\right)$. Since $M^{n} / M^{n+1}$ maps onto $\left(M^{n}+P\right) /\left(M^{n+1}+P\right)$, it follows that there is a surjective $k$-algebra homomorphism $\alpha: \operatorname{gr}(R) \rightarrow \operatorname{gr}(A)$.

We claim that $\alpha(X)=\alpha(Y)=0$. Now,

$$
\alpha(X)=\alpha\left(x+M^{2}\right)=x+\left(M^{2}+P\right) \in(M+P) /\left(M^{2}+P\right)
$$

and sinilarly, $\alpha(Y)=y+\left(M^{2}+P\right)$. Thus, the claim will follow if $x, y \in M^{2}+P$. We shall prove this next (showing also that $x \in M^{3}+P$ ).

If $x \in P$, then $y^{3}=x^{2}-f \in P+P=P$, whence $y \in P \subset M^{2}+P$. Similarly, $y \in P$ implies $x \in P$. Thus, without loss of generality, neither $x$ nor $y$ is in $P$. Let $v$ be a (discrete rank 1) valuation with value group $\mathbb{Z}$ and valuation ring $A$. Since $x+P$ and $y+P$ are nonzero elements of $N$, both $v(x+P)$ and $v(y+P)$ are positive integers. Now, applying $v$ to the equation $(x+P)^{2}=(y+P)^{3}$ [which holds since $f \in P$ ] yields $2 v(x+P)=3 v(y+P)$. Since 2 and 3 are relatively prime, $v(x+P)$ is an integral multiple of 3 , and $v(y+P)$ is an integral multiple of 2. In particular, $v(x+P) \geqslant 3$ and $v(y+P) \geqslant 2$. Thus,

$$
x+P \in N^{3}=(M / P)^{3}=\left(M^{3}+P\right) / P
$$


and, similarly, $y+P \in\left(M^{2}+P\right) / P$. Hence, $x \in M^{3}+P$ and $y \in M^{2}+P$, as asserted. This establishes that $\alpha(X)=\alpha(Y)=0$.

Next, consider any $\xi \in \operatorname{gr}(R)$. Since $\xi$ is integral over $k[X, Y]$,

$$
\xi^{n}+g_{1} \xi^{n-1}+\cdots+g_{n-1} \xi+g_{n}=0
$$

for some $g_{1}, \ldots, g_{n} \in k[X, Y]$. Apply the $k$-algebra homomorphism $\alpha$ to the displayed integrality equation. The result is

$$
\alpha(\xi)^{n}+\alpha\left(g_{1}\right) \alpha(\xi)^{n-1}+\cdots+\alpha\left(g_{n}\right)=0
$$

Now, since $\alpha(X)$ and $\alpha(Y)$ are both 0 , it follows that $\alpha\left(g_{i}\right) \in k$ for all $i=1, \ldots, n$. Hence, $\alpha(\xi)$ is algebraic over $k$, for all $\xi \in \operatorname{gr}(R)$. Since $\alpha$ is surjective, $\operatorname{gr}(A)$ is algebraic over $k$. Since $\operatorname{gr}(A) \cong k[T]$, it follows that $k[T]$ is algebraic over $k$. But $T$ is transcendental over $k$, and so we have produced the desired contradiction.

Proof OF $\left(^{*}\right)$ IF $k$ IS FINITE: Deny. Then, just as in the preceding proof, if $P \in \operatorname{Spec}(R)$ has height 1 , the inclusions $M^{n} \rightarrow M^{n}+P$ induce a surjective $k$-algebra homomorphism $\operatorname{gr}(R) \rightarrow \operatorname{gr}(R / P) \cong k[T]$. The kernel of this homomorphism is a nonmaximal prime ideal of $\operatorname{gr}(R)$. This kernel, which will be denoted by $\operatorname{gr}(R, P)$, is

$$
\operatorname{gr}(R, P)=\bigoplus_{n=1}^{\infty}\left(M^{n} \cap\left(M^{n+1}+P\right)\right) / M^{n+1}
$$

which, by an easy calculation, can also be written as

$$
\operatorname{gr}(R, P)=\bigoplus_{n=1}^{\infty}\left(\left(M^{n} \cap P\right)+M^{n+1}\right) / M^{n+1}
$$

Using either description, we see that $\operatorname{gr}(R, P)$ is a homogeneous ideal of $\operatorname{gr}(R)$.

Let $V=\{\operatorname{gr}(R, P): P \in \operatorname{Spec}(R), h t(P)=1\}$ and $W=\left\{\left(P+M^{2}\right) / M^{2}: P \in\right.$ $\operatorname{Spec}(R), h t(P)=1\}$. Note that $M / M^{2}$ is a finite dimensional $k$-space. Hence-and this is where we use the hypothesis that $k$ is finite $-M / M^{2}$ is a finite set. Therefore, so is $W$. We claim that, in fact, $V$ is a finite set. To show this, it suffices to prove that the funciton $\beta: V \rightarrow W$, given by $\beta(\operatorname{gr}(R, P))=\left(P+M^{2}\right) / M^{2}$ for all $P \in \operatorname{Spec}(R)$ with $h t(P)=1$, is an injection.

We shall show, in fact, that if $P \in \operatorname{Spec}(R)$ with $h t(P)=1$, then

$$
\left(\left(M^{n} \cap P\right)+M^{n+1}\right) / M^{n+1}=\left(\left(P+M^{2}\right) / M^{2}\right)\left(M^{n-1} / M^{n}\right)
$$

for each $n \geqslant 1$. One inclusion is evident. For the other, we shall show that

$$
M^{n} \cap P \subset P M^{n-1}
$$


for each $n \geqslant 1$. (Notice, for $n=1$, that this is simply $P \subset P$.) Since $R / P$ is a DVR, its maximal ideal is principal and nonzero. Thus, $M / P=(R / P)(\pi+P)$ for some $\pi \in M / P$; in particular, $M=R \pi+P$.

Consider $0 \neq p \in M^{n} \cap P$. Then $p$ is the sum of finitely many terms of the form $b_{i}=a_{i 1} \ldots a_{i n}$, with each $a_{i j} \in M$. Write $a_{i j}=r_{i j} \pi+p_{i j}$, with $r_{i j} \in R$, $p_{i j} \in P$. Expanding $b_{i}$, we find $b_{i}=r_{i 1} \ldots r_{i n} \pi^{n}+q_{i}$, where $q_{i} \in P M^{n-1}$. Thus, $p=\sum b_{i}=r \pi^{n}+q$, for some $r \in R, q \in P M^{n-1}$. It will suffice to prove that $r \in P$ (for then $p \in P M^{n}+P M^{n-1}=P M^{n-1}$ ). For this, notice that

$$
r \pi^{n}=p-q \in P+P=P,
$$

although $\pi \notin P$. Since $P$ is a prime ideal, it follows that $r \in P$, as desired. This concludes the proof that $\beta$ is an injection. (Notice that this part of the proof did not use the fact that $k$ is finite.) Now, since $k$ is finite, we have seen how the injectivity of $\beta$ assures us that $V$ has only finitely many, say $e$, elements. It will be convenient to write $V=\left\{\operatorname{gr}\left(R, P_{1}\right), \ldots, \operatorname{gr}\left(R, P_{\mathrm{e}}\right)\right\}$.

Consider $I=\bigoplus_{n=1}^{\infty} M^{n} / M^{n+1}$. This is a homogeneous maximal ideal of $\operatorname{gr}(R)$ which properly contains each $\operatorname{gr}\left(R, P_{i}\right)$. By prime avoidence lemma [11, Theorem 81], $I$ is not contained in $\operatorname{Ugr}\left(R, P_{i}\right)$. Since $I$ is homogeneous, there exist $d \geqslant 1$ and $F \in M^{d} / M^{d+1}$ such that $F \notin \operatorname{gr}(R, P)$, for each $P \in \operatorname{Spec}(R)$ with $h t(P)=1$.

Choose a coset representative, $f$, of $F$; that is, $f \in M^{d}$ and $F=f+M^{d+1}$. Since $F \neq 0$, we have $f \notin M^{d+1}$, and so $f \neq 0$. Thus, by the principal ideal theorem, if $Q \in \operatorname{Spec}(R)$ is chosen minimal over $R f$, we have $h t(Q)=1$. By the preceding paragraph, $F \notin \operatorname{gr}(R, Q)$. In particular, $F \notin\left(\left(M^{d} \cap Q\right)+M^{d+1}\right) / M^{d+1}$. This means that $f \notin\left(M^{d} \cap Q\right)+M^{d+1}$. However, $f \in M^{d} \cap Q$, and so we have produced the desired contradiction.

The proof of Theorem 3.1 is now complete.

\section{GoING-DOWN DOMAINS AND CPI-EXTENSIONS}

We begin by recalling some definitions and facts. Let $R$ be a domain. As in [4], $R$ is said to be a going-down domain in case $R \subset T$ satisfies GD (the going-down property) for each overring $T$ of $R$. Being a going-down domain is a local property. We say $R$ is divided in case $P=P R_{P}$ for each $P \in \operatorname{Spec}(R)$. If $R$ is divided, then $R$ is a quasilocal going-down domain [5, Proposition 2.1]. The converse is false in general [5, Example 2.9], but valid for $R$ seminormal [5, Corollary 2.6]. In particular, if $R$ is integrally closed, then $R$ is a going-down domain if and only if $R_{M}$ is a divided domain for each $M \in \operatorname{Max}(R)$ (see also [12, Corollary 11]).

We next use Proposition 2.3 to give two proofs (one using pullbacks, the other without) of the following main result. 
Theorem 4.1. If $R$ is an integrally closed going-down domain, then $R / P$ is integrally closed for each $P \in \operatorname{Spec}(R)$. (In other words, a going-down domain is in $\underline{C}$ if and only if $R$ is integrally closed.)

Proof: By the above comments, the hypotheses are preserved under localisation. Thus, by Proposition 2.1(a), we may assume that $R$ is quasilocal and hence, by the above comments, that $R$ is divided.

Our first proof uses Proposition 2.3(a). (Thus, by appeal to either [10] or [8], it uses pullbacks.) Accordingly, we are to show that $D=R+P R_{P}$ is integrally closed for each $P \in \operatorname{Spec}(R)$. However, since $R$ is divided, $P R_{P}=P$, whence $D=R$, which is integrally closed by hypothesis.

Our second proof uses the criterion in Proposition 2.3(b)(v). (Thus it avoids explicit use of pullbacks.) Suppose

$$
a=p\left(a^{-1}\right)^{n-1}-b\left(\sum_{i=0}^{n-1} r_{i}\left(b a^{-1}\right)^{n-1-i}\right)
$$

for some $P \in \operatorname{Spec}(R) ; p \in P ; a, b \in R \backslash P$; and $r_{0}, \ldots r_{n-1} \in R$. Since $a \notin P$, $a^{n-1} \notin P$ and $p\left(a^{-1}\right)^{n-1} \in P R_{P}=P$. Hence, the displayed equation yields $a \in(P, b)$, the desired contradiction.

Remark 4.2. (i) Theorem 4.1 leads to a new proof of the motivating result that any factor domain of a Prüfer domain is itself a Prüfer domain. For this we need to recall three facts:

(a) [5, Remark 2.11 or Remark 3.2(a)(b)]: each factor domain of a going-down domain is a going-down domain;

(b) [3, Corollary 4]: a domain is a Prüfer domain if and only if it is an integrally closed finite-conductor going-down domain;

(c) [6, Lemma 3.8]: if $D$ is a finite-conductor domain and $Q=Q D_{Q} \in \operatorname{Spec}(D)$, then $D / Q$ is a finite-conductor domain.

For the proof, let $R$ be a Prüfer domain and $P \in \operatorname{Spec}(R)$. We shall use (b) to show that $T=R / P$ is Prüfer. By Theorem 4.1 and (a), we need only show that $T$ is finite-conductor. Now, being finite-conductor is a local property, so it remains only to prove $T_{M / P} \cong R_{M} / P R_{M}$ is finite-conductor for each maximal ideal $M$ containing $P$. Since $R_{M}$ is a valuation domain, (c) evidently applies, with $D=R_{M}$ and $Q=P R_{M}$.

(ii) For each $n \geqslant 1$, there exists an $n$-dimensional going-down domain which is in $\underline{C}$ but is not a Prufer domain: combine Theorem 4.1 with [4, Corollary 4.4(i),(ii)].

Fact (a) recalled in Remark 4.2(i) asserted that the class of going-down domains is stable under formation of factor domains. In view of Theorem 4.1, it seems appropriate to identify some "nice"properties, where "nice" is somewhat weaker than "inte- 
grally closed," such that the class of "nice"going-down domains is stable under formation of factor domains. This will be done in Proposition 4.4, after recalling some "nice" background and developing a lemma about "nice"divided domains. Later, in Proposition 2.5(k),(l), we shall give related "nice"results without any going-down hypotheses.

Let $R$ be a domain with quotient field $K$, and let $S$ be a nonempty subset of positive integers. We say that $R$ is $S$-closed if $u \in K, u^{n} \in R$ for all $n \in S$ implies $u \in R$. For any finite $S$, it is easy to show that being $S$-closed is a local property. For any positive integer $n \geqslant 2, R$ is said to be $n$-root closed in case $R$ is $\{n\}$-closed. We say that $R$ is root closed in case $R$ is $n$-root closed for each $n \geqslant 2$. As in [9] for instance, we say that $R$ is seminormal in case $R$ is $\{2,3\}$-closed. In general, integrally closed $\Longrightarrow$ root closed $\Longrightarrow n$-root closed for some $n \geqslant 2 \Longrightarrow$ seminormal, with none of the implications being reversible. With this "nice" background in hand, we now give:

LEMma 4.3. Let $S$ be a nonempty subset of positive integers, $R$ a divided $S$ closed domain and $P \in \operatorname{Spec}(R)$. Then $R / P$ is $S$-closed.

Proof: Suppose $u \in R_{P} / P R_{P}=R_{P} / P$ satisfies $u^{n} \in R / P$ for each $n \in S$. Write $u=v+P$ for some $v \in R_{P}$. Thus, $v^{n} \in R+P=R$ for all $n \in S$. As $R$ is $S$-closed, $v \in R$, whence $u \in R / P$.

Proposition 4.4. Let $R$ be a going-down domain and $P \in \operatorname{Spec}(R)$. Then:

(a) if $R$ is $n$-root closed for some $n \geqslant 2$, then $R / P$ is $n$-root closed;

(b) if $R$ is root closed, then $R / P$ is root closed;

(c) if $R$ is seminormal, then $R / P$ is seminormal.

Proof: It is clear that (b) would follow from (a), and so it will be enough to prove (a) and (c). We give a unified proof for them, with $S$ meaning $\{n\}$ or $\{2,3\}$. Suppose $u \in R_{P} / P R_{P}$ satisfies $u^{k} \in R / P$ for all $k \in S$. Then, for each maximal ideal $M$ containing $P$ and each $k \in S$,

$$
u^{k} \in R / P \subset(R / P)_{M / P} \cong R_{M} / P R_{M} .
$$

By hypothesis, $R$ is $S$-closed (with $S$ either $\{n\}$ or $\{2,3\}$ ). Thus, by the above "nice"background, $R$ is seminormal. It follows that the quasilocal going-down domain $R_{M}$ is seminormal, and hence divided. As $R_{M}$ inherits the $S$-closed property from $R$, Lemma 4.3 yields that $R_{M} / P R_{M}$ is $S$-closed. Hence, so is $(R / P)_{M / P .}$. Thus $u \in \cap(R / P)_{M / P}=R / P$.

In Proposition 2.3(a), we saw how behaviour of the CPI-extensions of a domain $R$ could be used to characterise the condition $R \in \underline{C}$. Many related conditions considered 
in this paper admit similar characterisations. The next result collects many of these. For the sake of clarity and brevity, we avoid explicitly mentioning pullbacks and only sketch the simple proofs in Proposition 4.5.

Proposition 4.5. Let $R$ be a domain. Then:

(a) $R$ is a Prüfer domain $\Longleftrightarrow R+P R_{P}$ is a Prüfer domain for each $P \in$ $\operatorname{Spec}(R) \Longleftrightarrow R+M R_{M}$ is a Prüfer domain for each $M \in \operatorname{Max}(R) \Longleftrightarrow R / P$ is a Prüfer domain for each $P \in \operatorname{Spec}(R)$;

(b) $\operatorname{dim}(R) \leqslant 1 \Longleftrightarrow R+P R_{P}=R_{P}$ for each nonzero $P \in \operatorname{Spec}(R) \Longleftrightarrow$ $\operatorname{dim}\left(R+P R_{P}\right) \leqslant 1$ for each $P \in \operatorname{Spec}(R) \Longleftrightarrow \operatorname{dim}\left(R+M R_{M}\right) \leqslant 1$ for each $M \in$ $\operatorname{Max}(R) \Longleftrightarrow \operatorname{dim}(R / P) \leqslant 1$ for each $P \in \operatorname{Spec}(R)$;

(c) $R$ is a going-down domain $\Longleftrightarrow R+P R_{P}$ is a going-down domain for each $P \in S p e c(R) \Longleftrightarrow R+M R_{M}$ is a going-down domain for each $M \in M a x(R) \Longleftrightarrow R / P$ is a going down domain for each $P \in S p e c(R)$;

(d) $\operatorname{Spec}(R)$ is a tree $\Longleftrightarrow R \subset R+P R_{P}$ satisfies $G D$ for each $P \in \operatorname{Spec}(R) \Longleftrightarrow$ $S_{P e c}\left(R+P R_{P}\right)$ is a tree for each $P \in \operatorname{Spec}(R) \Longleftrightarrow \operatorname{Spec}\left(R+M R_{M}\right)$ is a tree for each $M \in M a x(R) \Longleftrightarrow S p e c(R / P)$ is a tree for each $P \in S p e c(R)$;

(e) $R$ is a valuation domain $\Longleftrightarrow R+P R_{P}$ is a valuation domain for each $P \in$ $\operatorname{Spec}(R) \Longleftrightarrow R / P$ is a valuation domain for each $P \in \operatorname{Spec}(R)$;

(f) $R$ is divided $\Longleftrightarrow R+P R_{P}$ is divided for each $P \in \operatorname{Spec}(R) \Longleftrightarrow R / P$ is divided for each $P \in \operatorname{Spec}(R)$;

(g) $R$ is a Dedekind domain $\Longleftrightarrow R+P R_{P}$ is a Dedekind domain for each $P \in$ $\operatorname{Spec}(R) \Longleftrightarrow R / P$ is a Dedekind domain for each $P \in \operatorname{Spec}(R)$;

(h) $R_{P}$ is divided for each $P \in S p e c(R) \Longleftrightarrow R_{M}$ is divided for each $M \in$ $\operatorname{Max}(R) \Longleftrightarrow R+P R_{P}$ is $R$-flat for each $P \in S p e c(R) \Longleftrightarrow R+P R_{P}$ is a ring of fractions of $R$ for each $P \in S p e c(R) \Longleftrightarrow$ each localisation of $R+P R_{P}$ is divided for each $P \in \operatorname{Spec}(R) \Longleftrightarrow$ each localisation of $R+M R_{M}$ is divided for each $M \in \operatorname{Max}(R)$;

(i) $R$ is a quasilocal going-down domain $\Longleftrightarrow R+R P_{P}$ is integral over $R$ for each $P \in \operatorname{Spec}(R)$;

(j) $R / P$ is integrally closed for each $P \in S p e c(R) \Longleftrightarrow R+R P_{P}$ is integrally closed for each $P \in \operatorname{Spec}(R)$;

(k) $R / P$ is (n-) root closed for each $P \in S p e c(R) \Longleftrightarrow R+P R_{P}$ is (n-) root closed for each $P \in \operatorname{Spec}(R)$;

(1) $R / P$ is seminormal for each $P \in \operatorname{Spec}(R) \Longleftrightarrow R+P R_{P}$ is seminormal for all $P \in \operatorname{Spec}(R)$.

Proof (sketch): (a) The key facts are that $R=R+0 R_{0} \cong R / 0$; if $P \subset Q$ are primes of $R$, the localisation of $R+P R_{P}$ at $Q+P R_{P}$ is $R_{Q}+P R_{P}[7$, Lenma 2.2(b)]; and any overring or factor domain of a Prüfer (respectively, valuation) domain 
is a Prüfer (respectively, valuation) domain.

(b) The first " $\Leftarrow$ " follows from the fact that if $P \subset Q$ are distinct primes of $R$, then $Q+P R_{P}$ is a prime of $R+P R_{P}$ which contracts to $Q$. For the first " $\Rightarrow$ ", take $b$ and $c$ in $R \backslash P$, use the maximality of $P$ to get $b \in(P, c)$, and conclude $b c^{-1} \in R+P R_{P}$. The next two equivalences follow from the bijection noted prior to Proposition 2.3.

(c) See [8, Proposition 4.6(6)] or [4, Proposition 3.2]; the localisation fact recalled in the proof of (a); and fact (a) recalled in Remark 4.2(i).

(d) The first equivalence is [7, Proposition 2.7]. For the next two equivalences, use the order-isomorphism recalled in the proof of $(b)$.

(e) Argue as in the proof of (a).

(f) See [7, Corollary 2.6]; the order-isomorphism recalled in the proof of (b); and [5, Lemma 2.2(c)].

(g) Argue as in the proof of $(\mathrm{a})$ and recall that $\operatorname{dim}(D) \leqslant 1$ for each Dedekind domain $D$.

(h) See [7, Theorem 2.4 and Corollary 2.6] and use the localisation fact recalled in the proof of (a).

(i) This is part of [6, Proposition 2.1].

(j) This is a restatement of Theorem 4.1.

(k) Assume first that each $R+P R_{P}$ is $(n-)$ root closed. Now if $u \in R_{P}$ is such that $\bar{u}=u+P R_{P}$ satisfies $\bar{u}^{n} \in R / P$, then $u^{n} \in R+P R_{P}$, whence $u \in R+P R_{P}$ and $\bar{u} \in R / P$. Thus, $R / P$ is (n-) root closed.

Conversely, assume each $R / P$ is $(n-)$ root closed. Putting $P=0$, we have $R$ is $(n-)$ root closed, and hence so is $R_{Q}$ for each $Q \in \operatorname{Spec}(R)$. Thus, if $u$ in the quotient field of $R$ satisfies $u^{n} \in R+Q R_{Q}$, we have $u \in R_{Q}$, so that $\bar{u}=u+Q R_{Q}$ in the quotient field of $R / Q$ satisfies $\bar{u}^{n} \in R / Q$. As $R / Q$ is $(n-)$ root closed, $\bar{u} \in R / Q$, whence $u \in R+Q R_{Q}$, as desired.

(l) Argue as in the proof of $(\mathrm{k})$, replacing $\{n\}$-closed condition with the $\{2,3\}$ closed condition.

Remark 4.6. (a) By the method of proof given for Proposition 4.5(k), (l), we have the following generalisation. Let $S$ be a finite nonempty set of positive integers and $R$ a domain. Then $R / P$ is $S$-closed for each $P \in \operatorname{Spec}(R) \Longleftrightarrow R+P R_{P}$ is $S$-closed for each $P \in \operatorname{Spec}(R)$.

(b) In contrast to the way in which Proposition 4.4 evolved from Theorem 4.1 by replacing "integrally closed" with "nice"weaker conditions, one may ask about analogues in which "integrally closed" is replaced by stronger conditions. We close by raising such a question. If $R$ is a completely integrally closed (cic) going-down domain and 
$P \in \operatorname{Spec}(R)$, must $R / P$ be cic? We doubt it, but note that the answer is affirmative if $R$ is quasilocal, by virtue of [5] and the following result. If a domain $R$ is cic and $P=P R_{P}$ is in $\operatorname{Spec}(R)$, then $R / P$ is cic.

\section{REFERENCES}

[1] M.B. Boisen Jr. and P.B. Sheldon, ' $C P I$ extensions: overrings of integral domains with special prime spectrums', Canad. J. Math. $2 \theta$ (1977), 722-737.

[2] N. Bourbaki, Algèbre Commutative, Chapitres 8 et 9 (Masson, Paris, 1983 ).

[3] D.E. Dobbs, 'On going-down for simple overrings', Proc. Amer, Math. Soc. 39 (1973), 515-519.

[4] D.E. Dobbs, 'On going-down for simple overrings II', Comm. Algebra 1 (1974), 439-458.

[5] D.E. Dobbs, 'Divided rings and going-down', Pacific J. Math. 67 (1976), 353-363.

[6] D.E. Dobbs, 'Coherence, ascent of going-down, and psuedo-valuation domains', Houston J. Math. 4 (1978), 551-567.

[7] D.E. Dobbs, 'On locally divided integral domains and CPI-overrings', Internat. J. Math. Math. Sci. 4 (1981), 119-135.

[8] M. Fontana, 'Topologically defined classes of commutative rings', Ann. Mat. Pura Appl. 123 (1980), 331-355.

[0] R. Gilmer and R.C. Heitmann, 'On Pic $(R[X])$ for $R$ seminormal', J. Pure Appl. Algebra 10 (1980), 251-257.

[10] B. Greenberg, 'Coherence in Cartesian squares', J. Algebra 50 (1978), 12-25.

[11] I. Kaplansky, Commutative Rings, rev. ed. (University of Chicago Press, Chicago, 1974).

[12] S. McAdam, 'Simple going-down', J. London Math. Soc. 13 (1976), 167-173.

[13] O. Zariski and P. Samuel, Commutative Algebra, Vol. I (Van Nostrand, Princeton, 1958).

[14] O. Zariski and P. Samuel, Commutative Algebra, Vol. II (Van Nostrand, Princeton, 1960).

Dr. V. Barucci

Dipartimento di Matematica

Università di Roma, "La Sapienza", 00185 Roma

Italy

Professor S.B. Mulay

Department of Mathematics

University of Tennessee

Knoxville, Tennessee 37996-1300

USA

\author{
Professor D.E. Dobbs \\ Department of Mathematics \\ University of Tennessee \\ Knoxville, Tennessee 37996-1300 \\ USA
}

